\title{
Game theoretic consideration of transgenic bacteria in the human gut microbiota as a pro- biotic prophylactic for metabolic syndrome
}

Ahmed M. Ibrahim ${ }^{1}$ and James Smith ${ }^{2}$

1 - 44 El-Geish St. Mansoura, Dakahlia, Egypt.

Email:wetawdt@gmail.com

2 - School of Food Science \& Nutrition, Faculty of Mathematics \& Physical Sciences, University of Leeds, LS2 9JT United Kingdom.

Email: j.smith252@leeds.ac.uk 


\begin{abstract}
A game theoretic treatment is introduced to explore the role of nutrition of the gut bacterial microflora as a potential pro-biotic therapy. Rational design of functional foods and nutraceuticals has far reaching public health and therapeutic benefits. Understanding ecological dynamics and how phenotypic manipulations of microbe-microbe interactions of the gut microbiota can provide direct health benefit is currently a fundamental question in bioengineering and more widely in the food, diet and health industries. This work considers a hypothetical adjustment of the microbiome by introducing a transgenic bacterial species that contributes to increased exposure of omega 3 fats in the gut by converting them from the omega 6 fats, dominant in the Western diet. The ratio of the two fats circulating in blood are risk markers, indicators of metabolic syndrome and related conditions. In this work, we consider nutritional exposure to a pro-biotic, a live culture of transgenic bacteria contributing omega 3 fats from omega 6 in the diet. Maintaining a long-term co-existence between native (indigenous) and transgenic bacteria is a challenge. Game theory is the appropriate tool for handling this conflict. The long-term co-existence is guaranteed if the two strains engage in the Snowdrift game. Our game theoretic treatment provides the basis of a model mechanism for prophylactic nutritional therapy for metabolic syndrome by the transgenic bacteria, providing support of indigenous gut microbiota and additional supplementation of a pro-biotic.
\end{abstract}

Keywords: game theory, snow drift game, prisoners' dilemma, nonlinear behaviour, gut microbiome, fat metabolism. 


\section{Introduction}

A game theoretic treatment is introduced to explore the role of nutrition of the gut bacterial microflora as a potential pro-biotic therapy for addressing the absorption of polyunsaturated fats (PUFAs) that are dominated by omega 6 (n6) class over the omega 3 (n3) class. A high n6 / $\mathrm{n} 3$ ratio is an indicator for high-risk metabolic syndrome, pre-type 2 diabetes and cardiovascular disease.

Metabolic syndrome is a cluster of strongly associated diseases including systemic cardiovascular disease, hypertension, osteoarthritis, stroke [1], lipodystrophies, pre-diabetes and obesity and a wide range of cancer types $[2,3]$. Long-term increased dietary exposure of $n 3$ and the increased $\mathrm{n} 3 / \mathrm{n} 6$ ratio of circulatory fats are considered to have protective benefits and to the colon $[4,5,6,7]$. However, the Western diet is typically dominated by n6 and depleted in n3, so-called fish oils but algal in origin. Exploitation of the dominant pool of n6 ingested from food to raise the fraction of bio-available n 3 counterpart species - lowering the $\mathrm{n} 6 / \mathrm{n} 3$ ratio - could form the basis of a cost effective functional food supplement and cost-effective therapy for high risk groups in the population. Considering that both $\mathrm{n} 6$ and $\mathrm{n} 3$ are essential nutrient fats, not intrinsically synthesized that have to be absorbed across the gut as free fatty acids and as components within in lipid molecules, the long-term adjustment of the $n 6 / n 3$ ratio, converting excess $n 6$ to $n 3$ within the gut would have far-reaching world-wide public health benefits.

This work considers a vignette, the conflict between indigenous gut bacterial colony and transgenic bacterial colony presented as a liveculture pro-biotic that provides raised n 3 nutritional exposure in the lumen of the gut. The introduced transgenic bacteria contributing to 
raised n3 expresses additional genes for enzymes involved in the conversion of excess n6. The genetic recombinant modifications are for example fatty acid desaturase (FADS) enzymes that convert n6 and illustrated in Figure 1 as red arrows, labelled respectively as $\Delta^{15,17} \mathrm{D}$, a desaturase converting linoleic acid (LA, C18:2n6) into alpha-linolenic acid (ALA, C18:3n3) and $\Delta^{14,17} \mathrm{D}$ a desaturase converting arachidonic acid (ARA, C20:4n6) into eicosapentaenoic acid (EPA, C20:5n3) and $\Delta^{16,19} \mathrm{D}$ a desaturase converting n6 DPA (Osbond acid, C22:5n6) to DHA(C22:6n3). In a recent review on polyunsaturated fatty acid (PUFA) biotechnology and desaturases, Lee et al [8] summarised reports in the literature that such equivalent n6 transformations are possible by selective $\omega 3$ Desaturase enzymes. Candidate genes for these enzymes are found in microbes and lower eukaryotes (algae) and would be exploitable for altering the $\mathrm{n} 6 / \mathrm{n} 3$ ratio in genetic recombinant bacteria. A discussion on the biochemical contributions and genetic re-engineering of $\omega 3$ Desaturases in addition to the details described in Figure 1, is beyond the scope of this article.

Importantly however, we generalise and assume here that the additional genes for the enzyme transformations (illustrated in red) are engineered and collectively provide a transgenic bacterial strain with a new metabolic phenotype with higher abundance of n3 fat-containing components compared to wild type strain. In our vignette, we consider a live culture of the transgenic bacterial colony introduced into the lumen of the gut as a pro-biotic, a functional food or rectal suppository. The application is analogous to the Caenorhabditis elegans fat-1 gene in Fat-1 mouse and livestock models $[9,10]$, the introduction of genetically transformed bacteria would certainly have potential to convert n6 to n 3 in situ. Candidate transgenic bacteria could be selected from symbiotic 
Bifidobacterium already found in the gut, conveniently transplanted or seeded into the intestine of patients $[11,12]$.

Recognising that the phenotype is stable, optimising inter-microbial interactions that govern the ecological niches between gut microbiota, would be the fundamental challenge for extending the longevity of genetically modified (gut) microbiota therapy [13].

The aim of this work is, therefore, to explore and discuss how a long-term co-existence between native wild type and transgenic bacteria could proceed. A game theoretical approach is ideal for this discussion because it can contribute to predicting the eventual stable endpoints of the populations and help in identifying the strategy (or set of strategies) that cannot be invaded by rare alternative strategies, called the evolutionarily stable strategy (ESS) [14]. 


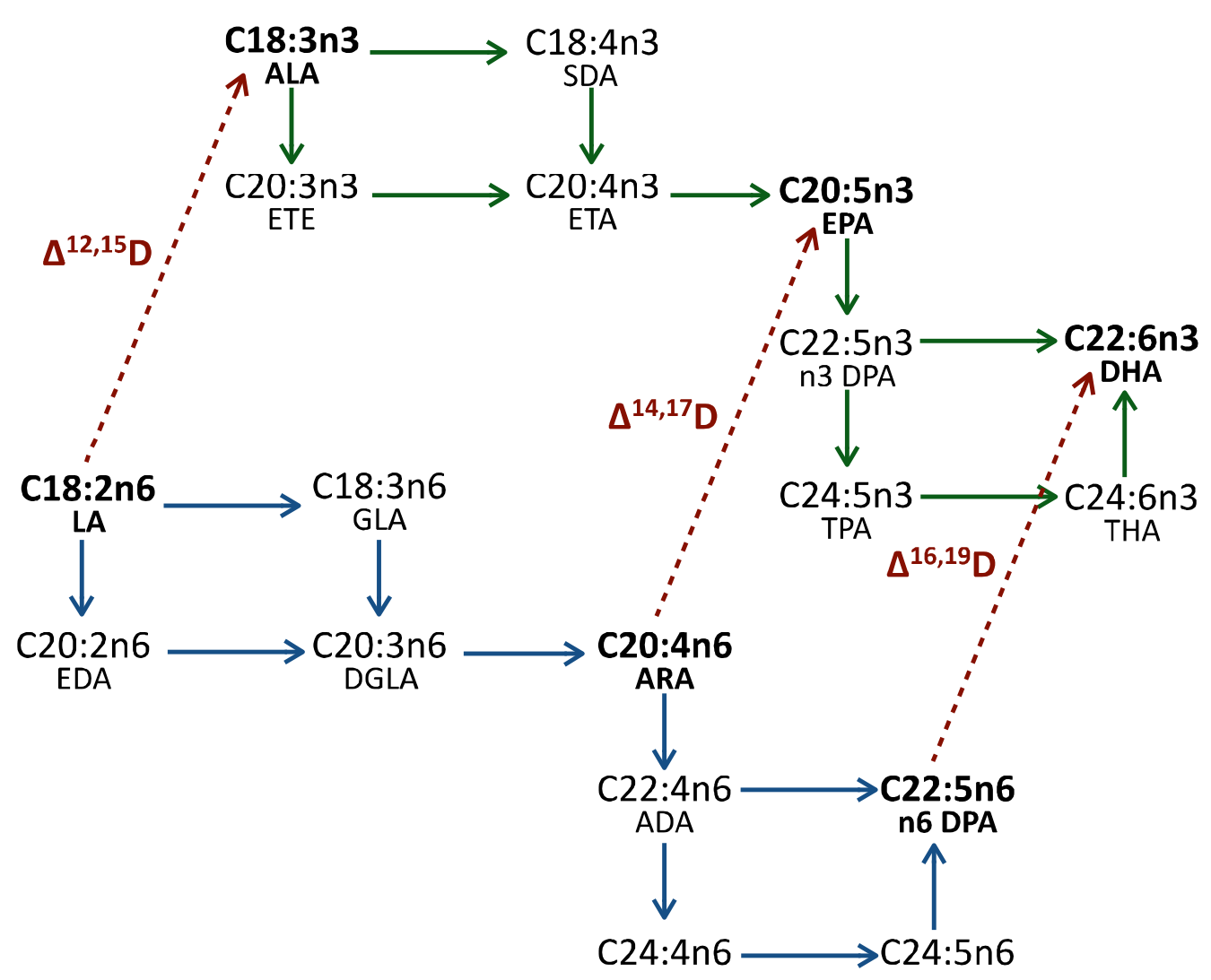

Figure 1. Proposed transgenic $\omega 3$ Desaturase enzyme transformations (dotted arrows in red) as the potential pro-biotic prophylactic therapy to change the naturally high n6/n3 ratio of polyunsaturated fats (PUFAs). The $\omega 3$ Desaturase enzymes would act by lowering the abundance of omega 6 PUFAs (n6, network in blue) and raise the abundance of omega 3 PUFAs (n3 network in green). The PUFAs are labelled here by their canonical numbering and labelling and by their abbreviations. The directed networks show the endogenous conversions of the PUFAs and include elongation (down arrows), beta-oxidation (up arrows) and desaturation (left to right arrows), assumed to exist in indigenous gut flora and fauna and in human physiology (further enzymatic details not shown). 


\section{Theoretical Treatment}

In the Snowdrift game (or Chicken game, Hawk-Dove game) there is a mixed evolutionarily stable strategy (ESS), where it is always advantageous to adopt the opposite strategy of an opponent. Snowdrift (SD) is illustrated by a scenario, where two car drivers are trapped on either side of a snowdrift and have the options of staying in the car or removing snow from the drift from each of their sides. Allowing the opponent do all the work is the best (selfish) option, however if the opponent remains in their car it is better to shovel, lest no one gets home. Joint co-operation reduces the work load, ensuring that both can drive home.

Should both players co-operate $(\mathrm{C}, \mathrm{C})$ then they both receive the reward $(R)$ for co-operation. If both players defect $(\mathrm{D}, \mathrm{D})$, they both receive punishment $(P)$ for mutual defection. Should one defect while their opponent co-operates $(\mathrm{C}, \mathrm{D}$ or $\mathrm{D}, \mathrm{C})$, then the defector receives the "temptation payoff" $T$, while the co-operator receives the "sucker's payoff", $S$ (Table 1).

Table 1. The snowdrift game payoff matrix.

\begin{tabular}{r|cc} 
& $\begin{array}{c}\text { Opponent plays } \\
\text { Co-operator C }\end{array}$ & $\begin{array}{c}\text { Opponent plays } \\
\text { Defector D }\end{array}$ \\
\hline Focal player plays Co-operator C & $R$ & $S$ \\
Focal player plays Defector D & $T$ & $P$
\end{tabular}


In $\mathrm{SD}$, co-operation yields a benefit $b$ to the co-operator as well as to the opposing player and incurs a cost $c$ if the opponent defects, but only a cost $c / 2$ if the opponent co-operates, where $b>c>0$. The defector receives the payoff $b$ and mutual defection has no net cost nor net benefit, where, $T=b, R=b-c / 2, S=b-c$ and $P=0$ (see Table 2).

Table 2. The fitness payoff structure of the snowdrift game.

\begin{tabular}{r|cc} 
& $\begin{array}{l}\text { Opponent plays } \\
\text { Co-operator }\end{array}$ & $\begin{array}{l}\text { Opponent plays } \\
\text { Defector }\end{array}$ \\
\hline Focal player plays Co-operator & $b-c / 2$ & $b-c$ \\
Focal player plays Defector & $b$ & 0
\end{tabular}

This leads to the characteristic payoff ranking, $T>R>S>P$. Therefore, it is always better to play as a co-operator when the opponent defects (the probability of encountering a defection increases when defectors are common). Thus, co-operation here is an ESS strategy and co-operators cannot be invaded $(S>P)$. However, it is also always better to play as a defector when the opponent co-operates (as the probability of encountering co-operation increases when co-operators are common). Thus, defection here is an ESS strategy and defections cannot be invaded $(T>R)$. Consequently, defectors and co-operators will co-exist. Hence, for getting long-term coexistence between wild type (indigenous) and transgenic bacteria without either one out-competing the other, the two strains would have to engage in an SD interaction.

In our potential pro-biotic prophylactic therapy, the thermodynamic cost of expressing and producing additional $\omega 3$ Desaturases could decrease the fitness of the genetically engineered (transgenic) bacteria, resulting in 
their being out-competed by the indigenous gut bacteria (such as Bifidobacterium, Akkermansia muciniphila, and Lactobacillus [15]). The presence of the new $\omega 3$ Desaturases, however, could also confer a phenotypic advantage. For example, an increase in the n 3 would alter the phospholipid components and functionality of the cell wall and its component LPS also known as lipoglycan endotoxin that locally suppresses neighbouring indigenous gut bacteria. Therefore, if the transgenic bacteria were indeed from strain of Bifidobacterium or Lactobacillus, there could be an adaptive phenotypic advantage when expressing more n3. Although, there would be an energetic cost in producing the new desaturases, there could also be a growth advantage as n3-containing lipid components become more abundant. The transgenic bacteria would play as a co-operator and the wild-type (indigenous) would be the defector. We assume that the indigenous bacteria will not out-compete the transgenic bacteria as in the case of the PD interaction but instead co-exist in the Snow Drift (SD) interaction. We assume that the producers (transgenic bacteria) can benefit from increased n3containing lipid components before the indigenous bacteria can and that there is net gain from expressing the additional $\omega 3$ Desaturases. The interaction would convert to the PD interaction [16] if there is a net (energetic or phenotypic disadvantage) in expressing the additional $\omega 3$ Desaturases, where $2 b>c>b$ instead of $b>c>0$.

The situation above is similar to the competition between the wild type co-operator cells of yeast and the cheater (defector) strains (see, Gore et al [17]). Here, co-operator cells produce invertase an enzyme to hydrolyse disaccharide sugars into single sugar residues then $99 \%$ of the these spread to and exploited by neighbouring non-producer cells (the cheaters). Yeast cells are known to co-exist and show SD interaction and 
not PD interaction because the producers reap the profits before the other surrounding cells [17]. Consequently, the SD interactions ensures a longer-term co-existence between the two strains.

Consider a population of $N$ members where the fraction of the cooperators is $x$ and the fraction of the defectors is $1-x$; the parameters $T$, $R, S$ and $P$ in (Table 2) can be reduced to a single parameter if $R=1$ and $P=0$. The resulting cost-to-benefit ratio $r=c /(2 b-c)$ and this yields, $T=1+r$ and $S=1-r$, where $0<r<1.0$.

Table 3. The rescaled payoff matrix.

\begin{tabular}{r|cc} 
& $\begin{array}{l}\text { Opponent plays } \\
\text { Co-operator }\end{array}$ & $\begin{array}{l}\text { Opponent plays } \\
\text { Defector }\end{array}$ \\
\hline Focal player plays Co-operator & 1 & $1-r$ \\
Focal player plays Defector & $1+r$ & 0
\end{tabular}

The equilibrium fractions, from a well-mixed population of co-operators and defectors can be derived using the replicator equation. Replicator dynamics describe the evolution of the frequencies of strategies in a population (from Hofbaueret et al [18]), where

$$
\frac{d x}{d t}=x(1-x)\left(P_{C}-P_{D}\right)
$$

$P_{C}$ denotes the average payoffs of co-operators,

$$
P_{C}=x R+(1-x) S
$$

and $P_{D}$ denotes the average payoffs of defectors,

$$
P_{D}=x T+(1-x) P
$$

Equation (1) has three equilibrium fixed points $x_{1}=0, x_{2}=1$ and $x_{3}$ where $P_{C}=P_{D}$. The third is a stable equilibrium fixed point because the system 
always returns to it after perturbation, while, the system would move away from the unstable fixed points $x_{1}$ and $x_{2}$ after perturbation.

Substituting into Equations (2) and (3) where $R=1$ and $P=0$

$$
x+(1-x) S=x T
$$

and this leads to

$$
x_{3}=\frac{S}{(T+S-1)}
$$

Substituting into the rescaled payoff matrix (Table 3),

$$
x_{3}=1-r
$$

Therefore, the stable equilibrium of the fraction of co-operators will be

$$
x_{3}=1-\frac{c}{(2 b-c)}
$$

It is interesting that the results of the continuous snowdrift game (CSD) are compatible with the one-shot traditional snowdrift game [19]. The time evolution of the frequency of the co-operators could be shown by numerical simulation at different values of $r$, where $0<r<1$. For instance, if $r=0.7, T=1.7, S=0.3, x_{3}=0.3$. Co-operators and defectors therefore can co-exist in the same population in a stable state of $30 \%$ and $70 \%$ respectively (Figure 2 ). 


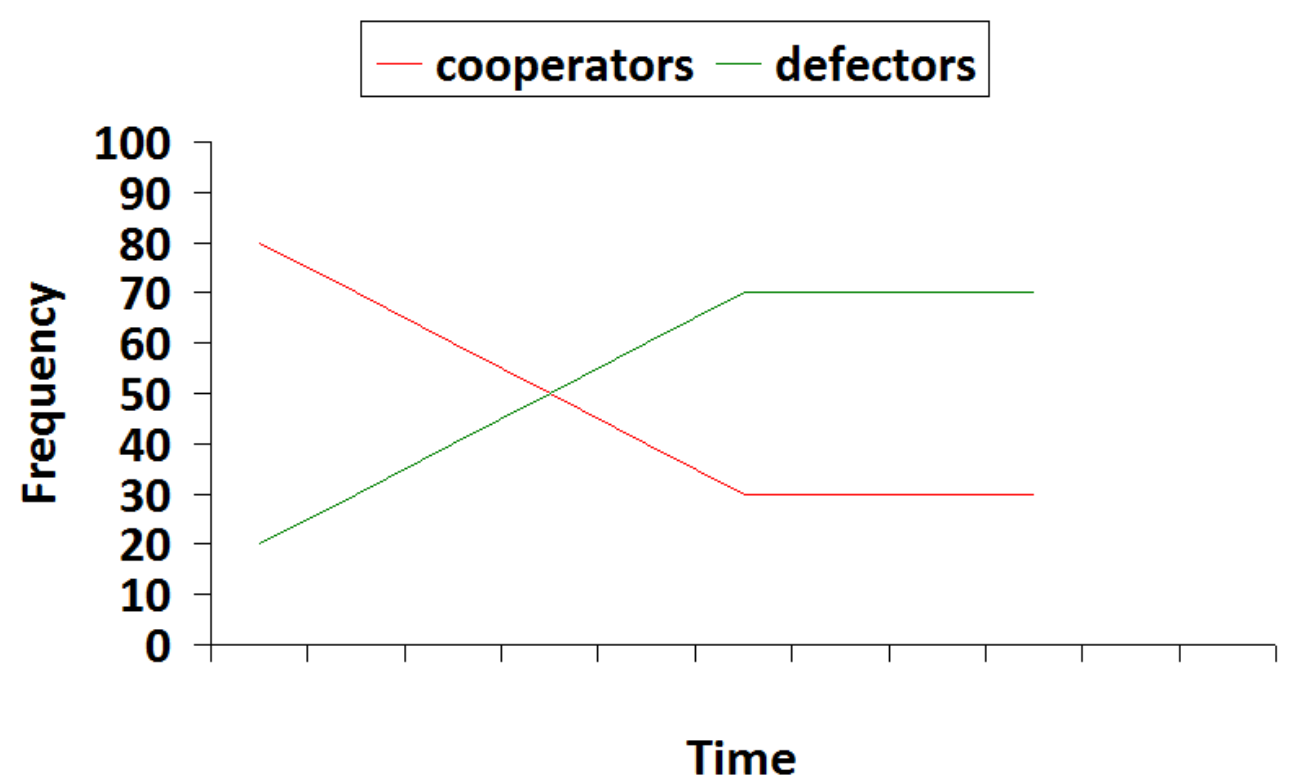

Figure 2. When $r=0.7$ the equilibrium frequency of the co-operators according to the replicator equation will be 0.30 . The stable fraction in the population will be 0.30 cooperation and 0.70 defection, respectively.

The interaction (game) will also be PD instead of SD if the co-operation results in a benefit $b$ to the opposing player, but incurs a cost $c$ to the cooperator (where $b>c>0$ ), the defector receives the payoff $b$, and mutual defection has no costs or benefits. The payoff rank will be $T>R>P>S$, where

$T=b, R=b-c, P=0$ and $S=-c$ (Table 4$)$.

In PD, defection is a pure ESS unbeatable strategy. Therefore, it is always better to adopt the defection strategy regardless of the strategy that the opponent will adopt. Hence defectors will drive co-operators to extinction. 
Table 4. Fitness payoff of the prisoners' dilemma.

\begin{tabular}{r|cc} 
& $\begin{array}{c}\text { Opponent plays } \\
\text { Co-operator }\end{array}$ & $\begin{array}{c}\text { Opponent plays } \\
\text { Defector }\end{array}$ \\
\hline Focal player plays Co-operator & $b-c$ & $-c$ \\
Focal player plays Defector & $b$ & 0
\end{tabular}

Clearly, there is an empirical problem in determining the payoff structure in social interactions between microbe colonies in nature. Consequently, it is difficult to distinguish experimentally between prisoner's dilemma and snowdrift interactions. Interestingly, work by Fu et al [20] suggest an indicator that could solve this problem - Spatial lattice models of macroscopic patterning, exhibiting critical phenomena, reveal that snowdrift interactions have filament-like dendritic clusters, showing percolation behaviour, whereas prisoner's dilemma interactions reveal compact isolated clusters, below a critical propagation threshold.

As an aside, the ability to distinguish emergent patterns of microbial interactions would provide the basis of pattern recognition for colony detection, from faecal microbiology cultures or during endoscopic procedures (for example, with engineered Green Fluorescence Protein in transgenic bacteria [21], borrowing methodological aspects of Borisova et $a l[22]$ in the clinic, cultured colony detection $[23,24]$ in the laboratory and colony detection summarised by Hickley et al [25]) with which to visualise the spatial-temporal distribution of the most beneficial and effective densities of the probiotic.

After ensuring the outcome of SD, the transgenic bacteria colonies can transmit their effect by providing the omega (n3) fat components to the gut wall. Snowdrift is a two-person game however and realistic interactions are multi-person. We therefore consider Zheng's model of 
evolutionary snowdrift games with N-person interactions (NESG) [26].

According to the NESG model, in infinite populations without assortments $[26,27]$ the payoff value for a co-operator is

$$
P_{C}(n)=b-\frac{c}{n}, \text { for } n \in[1, N]
$$

and the payoff value for a defector is

$$
P_{D}(n)= \begin{cases}0 & n=0 \\ b & n \in[1, N-1]\end{cases}
$$

where, $n$ is the number of co-operators, $N$ represents the number of group members and the benefit $(b)$ and cost $(c)$ of the collective work. The average fitness of the co-operators and defectors, are respectively,

$$
f_{C}=\sum_{j=0}^{N-1}\left(\begin{array}{c}
N-1 \\
j
\end{array}\right) x^{j}(1-x)^{N-1-j} P_{C}(j+1),
$$

and

$$
f_{D}=\sum_{j=0}^{N-1}\left(\begin{array}{c}
N-1 \\
j
\end{array}\right) x^{j}(1-x)^{N-1-j} P_{D}(j) .
$$

Again, both $x$ and $(1-x)$ are the fractions of co-operators and defectors respectively in $N$ members but importantly, $j$ is the number of cooperators in the group.

The average fitness of the player is

$$
\bar{f}(t)=x(t) f_{C}(t)+(1-x(t)) f_{D}(t) .
$$

The time evolution of the frequency of co-operation $x(\mathrm{t})$ is governed by the following differential equation,

$$
\frac{d x}{d t}=x\left(f_{C}-\bar{f}\right)
$$

from (1) the replicator equation of frequency of co-operation $x(\mathrm{t})$ becomes

$$
\frac{d x}{d t}=x(1-x)\left(f_{C}-f_{D}\right) .
$$


The stable fixed point or the stable equilibrium frequency of cooperation $x^{*}$ satisfies

$$
f_{C}\left(x^{*}\right)=f_{D}\left(x^{*}\right)
$$

and the stable equilibrium of co-operators can be described by an $\mathrm{N}$-thorder equation, Equation (16), where $r=c / b$ denote to the cost-tobenefit ratio and $x^{*}$ the frequency of co-operation in a group of $N$ members.

$r\left(1-x^{*}\right)^{N}+N x^{*}\left(1-x^{*}\right)^{N-1}-r=0$. 


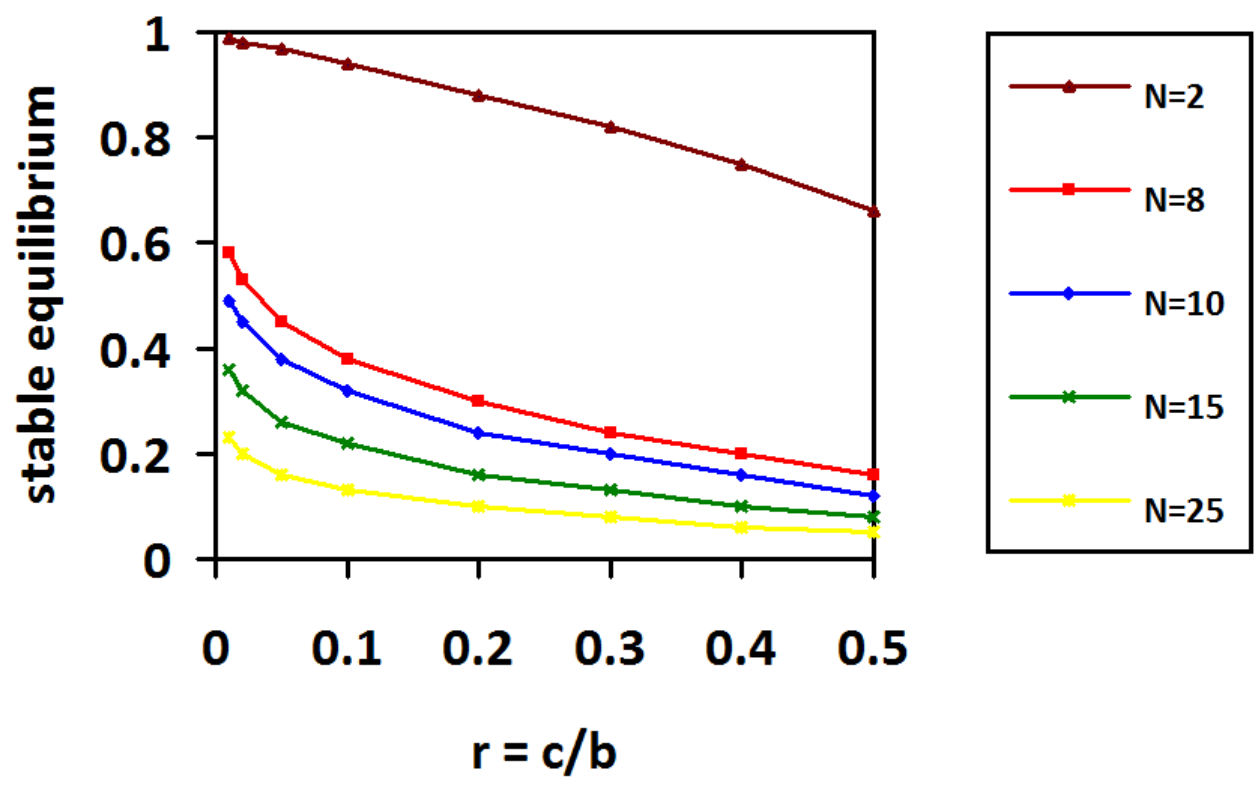

Figure 3. Numerical simulation for Equation (16) illustrating the stable equilibrium of co-operators at different frequencies of low ratios of cost-benefit using random sampling for $N=2,8,10,15$ and 25 . 


\section{Discussion}

The natural strains of gut microbiota (e.g. Bifidobacterium, Akkermansia muciniphila, and Lactobacillus) have therapeutic effects in a broad range of diseases as probiotics [reviewed in 28, 29, 30] such as inhibiting the growth of harmful bacteria, reducing cholesterol levels, synthesizing vitamins, and treatment of metabolic disorders [31]. Therefore, intake of the genetically modified gut micrbiota as probiotics is considered per se a therapeutic mechanism for metabolic syndrome. This feature will not be lost in our model; rather it is supported in an environment rich in n3. Therefore, modified strains expressing $\omega 3$ desaturases provide a model with multiple therapeutic mechanisms (Figure 4).

In general, the gram-positive bacteria may have some obstacles to secrete enzymes. Some studies have shown that some strains such as the nonmotile Bifidobacterium longum can perform this task [32]. Other studies have implicated Bifidobacterium pseudocatenulatum CECT 7765 strain with a significant therapeutic effect of reducing inflammation and metabolic endotoxemia [33]. Different Bifidobacterium species and strains may have different advantages. Hence, we should assume there is a trade-off between all appropriate strains. 


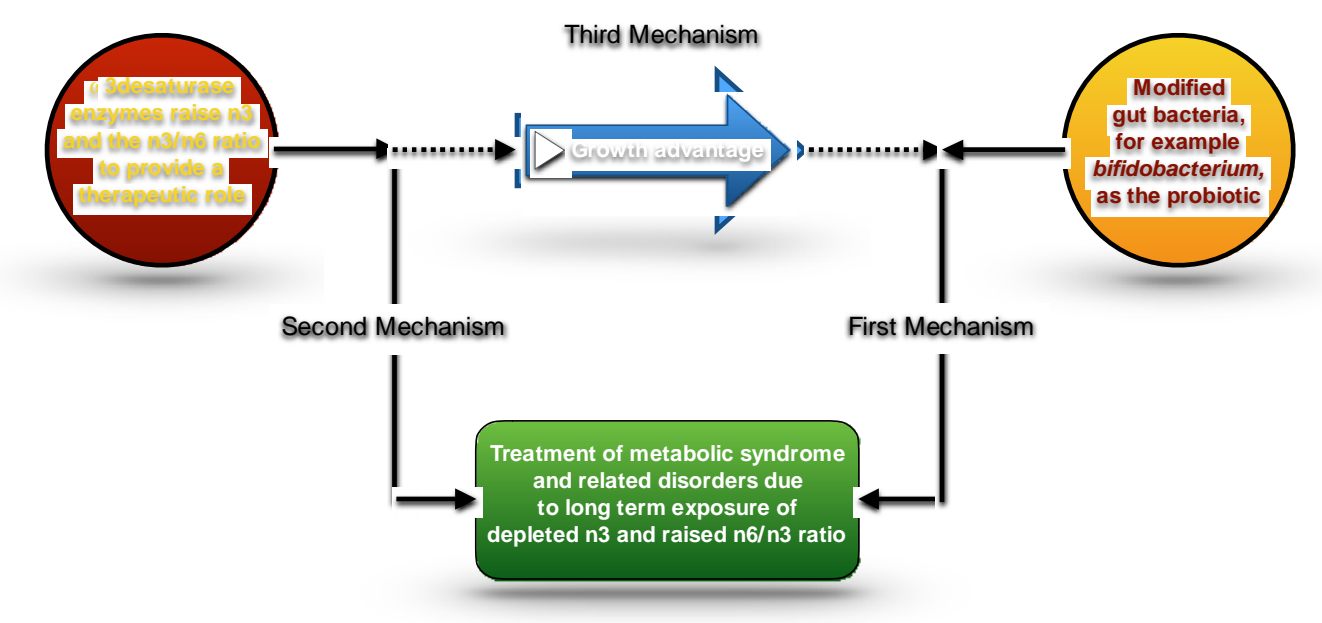

Figure 4. In this model, there are three synergistic mechanisms for treatment of metabolic syndrome and related disorders.

\subsection{Limitations}

We used the traditional models of SD and NSD in infinite unstructured populations to shed the light on the possibility of long-term co-existence between the wild type and the transgenic bacteria that in turn are appropriate for prophylactic therapy.

The model does not consider the dynamic of the whole gut species but focuses only on the dynamic between the indigenous and transgenic strain. The assumption in the model is that there are just two strategies interacting with each other, is oversimplified and neglects the contribution of three strategy games [34] (e.g. Rock-Scissors-Paper game) where there are individuals that neither reap benefit nor incur cost. Furthermore, the model does not identify the thresholds of co-operators in the N-person snowdrift game, namely the minimum number of coordinated co-operators required to build the public good [27] and the higher threshold of co-operators beyond which additional co-operators 
produces no more net benefit [34]. The model also does not consider the evolutionary dynamics of N-person snowdrift game in both structured and finite populations $[35,36]$.

Although the snowdrift game will lead to co-existence between cooperators and defectors, the spatial structure may suppress the cooperation if it is not dynamic, contributing to the mixing gut microbiota populations $[37,38]$. Therefore, co-operators would not be able to develop compact clusters because such patterns increase the proportion of interacting with only other co-operators. Hence, it becomes better to be a defector. Thereby, defectors penetrate co-operators patches and cooperators may extinct if adopt limited dispersal dynamics.

Many theoretical and empirical studies ensure that [39, 40, 41] plasmids which carry cooperative trait can promote co-operation with-in spatial structured patches. In particular, in environmental conditions such as gastrointestinal where the abundance of nutrients [42], by increasing relatedness and homogeneity of the patches via infect the defectors invader with the cooperative traits in the absent of selection for these cooperative traits, provided the null plasmid which carries defection traits was absent or does not have a higher rate of transfer. Consequently, if the rate of infection of defector-invaders by a cooperative mobile element was greater than the rate of colonization of co-operator patches by defectors, the harmful effect of spatial structure on SD game will be averted. Thus, for exceeding the problem of spatial structure we suggest that the cooperative trait (the genes for the desaturases) should be loaded on plasmid. 


\subsection{Future work}

From an evolutionary ecological perspective, there are many promising therapeutic applications that can be achieved (for examples, see [43, 44, $45,46,47])$. If by gaining more insight on heterogeneous microbial interactions and relating them to more sophisticated models of PD and SD-based interactions, critical traits can potentially be eliminated or added to selectively target different patient cohorts.

Subsequently, manipulate the fate of the pathogenic agents leading it to extinction by engaging it in Prisoner's dilemma $[47,48,61]$ or acquire novel benefits by engaging the engineered beneficial agents in snodrift as in our present work.

All above models address the unconditional deterministic cooperationldefection strategies rather than conditional probabilistic memory-one strategies (e.g. Tit-for-tat (TFT), Win-stay lose-shift (WSLS) etc.). A recent revolutionary discovery by Press and Dyson [49, $50]$ is the conditional zero-determinant (ZD) strategies that allow a player to unilaterally pin the opponent payoff at a certain value or dictate a linear relationship between the own payoff and the opponent payoff, irrespective of the opponent decisions and irrespective of the group size in multiplayer games. ZD strategies are significant powerful long-term control strategies. Interestingly, these strategies are not confined to the iterated prisoner's dilemma or two player games but extended to all public goods games including symmetric social dilemmas [51, 53]. ZD strategies may be fair, extortion or generous.

Individuals adopting extortion ZD strategy reap an unfair share of the payoff from the opponent, on the other hand, they provide incentives to 
co-operate. Therefore, All-C (the unconditional deterministic strategy that always co-operates) is always the best response to an extortion strategy. Although ZD extortion strategies can dominate any co-operator evolutionary (non-sentient player who accede to extortioner's actions for maximizing his own score without trying to alter the extortion behaviour) opponent in head-to-head matches, it is still unstable evolutionary strategy as it doesn't act against itself due to mutual defection [53,54]. Therefore, it will win each battle but lose the whole war [55]. For turning it into a stable strategy, extortioners may need for additional mechanisms which enhance the outcomes of interactions between them such as impose players co-operate with themselves but not against the opponent as in coordination games which in turn requires a minimum amount of coordinators. This may be achieved by $i$ ) tag-based strategies [53] in which tags provide extra information for cooperating only with other extortioners. $i$ ) If the extortioners exist in one species which has the slower evolutionary rate of two different interacting species [54] (for example hosts and their symbionts) or two asymmetric classes of the same species.

Generous ZD strategies [56, 57, 58] (compliant strategies) co-operate with others to reap lower payoff than its co-operator co-player and forgive defectors or punish them mildly. A generous ZD strategy is always the best response to itself. Because the baseline payoff (the payoff that a player would get against himself) of compilers is the maximum possible payoff could be reaped in the population $(R)$ (no defecting strategy can yield a payoff higher than $\mathrm{R}$ when playing against a complier except in the presence of altruistic strategies) [58]. Generous ZD strategies can dominate any defector strategy in the long term even All-D (the unconditional deterministic strategy that always defect). However, 
unlikely extortioners generous ZD strategies are evolutionarily robust strategy (ERS) [59] and can replace all other classic IPD strategies (e.g. TFT, WSLS, All-D... etc.) and even extortion ZD strategies except in very small populations [60].

This is clearly could be a helpful tool for establishing more powerful cooperator transgenic organisms using for the same therapeutic purpose which we suggesting in this article. In general, selection disfavour zerodeterminant strategists in large populations [57]. Apparently ZD strategies play an important transient role in the population dynamics, it can be shown in the iterated prisoner's dilemma as the following cycle: both unconditional strategies All-C and All-D enforce linear relationship with a negative slope then the baseline payoff of the whole population decreases over time. Therefore, the population converts from cooperation to defection but extortioners can exist in All-D population by neutral drift then the population converts from defection to co-operation because both conditional strategies extortion ZD and generous ZD enforce linear relationship with a positive slope then the baseline payoff of the whole population increases over time. Eventually, altruistic strategies can invade generous ZD then All-D will rise again (in iterated snowdrift game the slope of All D is positive but the dynamics remain the same) [60].

If tag-based strategies or any other similar strategies succeed to convert extortion ZD to a stable strategy by altering the behaviour of the extortioners against themselves, the extortion ZD will not be anymore the extreme bottom edge of the baseline payoff of the whole population, also it will not be a memory-one strategy. Therefore, it could be used for a therapeutic purpose by outcompeting all of the other strategies, for 
instance, it can homogenize the colonies of cancer cells or viral strains in the patient. Therefore, it makes them an easy target for drugs or even further it may be invaded by transgenic All-D which imitating the tag [53], then the pathogenic population will be very week or even go extinct (This suggestion is the inverse pattern of our present article). 


\section{References}

1 - Smith, K. B., \& Smith, M. S., Obesity Statistics. Primary Care:

Clinics in Office Practice, 43(1), 121-135;

doi: 10.1016/j.pop.2015.10.005 (2016).

2 - García-Jiménez, C., Gutiérrez-Salmerón, M., Chocarro-Calvo, A., García-Martinez, J. M., Castaño, A., \& De la Vieja, A., From obesity to diabetes and cancer: epidemiological links and role of therapies.

British Journal of Cancer, 114(7), 716-722;

doi: 10.1038/bjc.2016.37 (2016).

3 - Wang, M., Hu, R.-Y., Wu, H.-B., Pan, J., Gong, W.-W., Guo, L.H., Zhong, J.-M., Fei, F.-R., and Yu, M., Cancer risk among patients with type 2 diabetes mellitus: a population-based prospective study in China. Scientific Reports. 5, 11503;

doi: 10.1038/srep11503 (2015).

4 - Kim, Y. S., Xun, P., Iribarren, C., Van Horn, L., Steffen, L., Daviglus, M. L., Siscovick, D. Liu, K and He, K., Intake of fish and long-chain omega-3 polyunsaturated fatty acids and incidence of metabolic syndrome among American young adults: a 25-year followup study. European Journal of Nutrition, 55(4), 1707-1716;

doi: 10.1007/s00394-015-0989-8. (2016).

5 - Tørris, C., Molin, M., and Småstuen, M. C., Fish consumption and its possible preventive role on the development and prevalence of metabolic syndrome - A systematic review. Diabetology \& Metabolic Syndrome, 6(1), 112;

doi: 10.1186/1758-5996-6-112 (2014).

6 - Masquio, D. C. L., de Piano-Ganen, A., Oyama, L. M., da Silveira Campos, R. M., Santamarina, A. B., de Souza, G. I. d. M. H., Gomes, A. D., Moreira, R. G., Corgosinho, F. C., Nascimento, C. M. O., Tock, L., Tufik, S., de Mello, M. T., and Dâmaso, A. R., The role of free fatty acids in the inflammatory and cardiometabolic profile in adolescents with metabolic syndrome engaged in interdisciplinary therapy. The Journal of Nutritional Biochemistry, 33, 136-144; doi: 10.1016/j.jnutbio.2016.03.017 (2016). 
7 - Barden, A. E., Mas, E., Croft, K. D., Phillips, M., and Mori, T. A., Specialized pro-resolving lipid mediators in humans with the metabolic syndrome after n-3 fatty acids and aspirin. The American Journal of Clinical Nutrition, 102(6), 1357-1364;

doi: 10.3945/ajen.115.116384 (2015).

8 - Lee, J., Lee, H., Kang, S., \& Park, W. Fatty Acid Desaturases, Polyunsaturated Fatty Acid Regulation, and Biotechnological Advances. Nutrients, 8(1), 23-13;

doi: 10.3390/nu8010023 (2016).

9 - Kang, J. X., Wang, J., Wu, L., and Kang, Z. B. Transgenic mice: fat- 1 mice convert n-6 to n-3 fatty acids. Nature, 427(6974), 50; doi: 10.1038/427504a (2004).

10 - Lai, L., Kang, J. X., Li, R., Wang, J., Witt, W. T., Yong, H. Y., Hao, Y., Wax, D. M., Murphy, C. N., Rieke, A., Samuel, M., Linville, M. L., Korte, S. W., Evans, R. W., Starzl, T. E., Prather, R. S., and Da, Y., Generation of cloned transgenic pigs rich in omega-3 fatty acids. Nature biotechnology, 24(4), 435-436; doi: 10.1038/nbt1198 (2006).

11- Braat, H., Rottiers, P., Hommes, D. W., Huyghebaert, N., Remaut, E., Remon, J. P., Deventer, S. J. H. V., Neirynck, S., Peppelenbosch, M. P., and Steidler, L., A phase I trial with transgenic bacteria expressing interleukin-10 in Crohn's disease. Clinical Gastroenterology and Hepatology, 4(6), 754-759; doi: 10.1016/j.cgh.2006.03.028 (2006).

12- Chen, Z., Guo, L., Zhang, Y., Walzem, R. L., Pendergast, J. S., Printz, R. L., Morris, L.C., Matafonova, E., Stien, X., Kang, L., McGuinness, O. P., Niswender, K. D., Davies, S. S., \& Coulon, D.. Incorporation of therapeutically modified bacteria into gut microbiota inhibits obesity. The Journal of clinical investigation, 124(8), 33913406; doi:10.1172/JCI72517 (2014).

13 - Holmes, E., Kinross, J., Gibson, G. R., Burcelin, R., Jia, W., Pettersson, S., \& Nicholson, J. K., Therapeutic modulation of microbiota-host metabolic interactions. Science Translational Medicine, 4(137), 137rv6-137rv6;

doi: 10.1126/scitranslmed.3004244 (2012). 
14 - McGill, B. J., \& Brown, J. S. (2007). Evolutionary game theory and adaptive dynamics of continuous traits. Annual Review of Ecology, Evolution, and Systematics, 38, 403-435;

doi: 10.1146/annurev.ecolsys.36.091704.175517 (2007).

15 - Kaliannan, K., Wang, B., Li, X. Y., Kim, K. J., \& Kang, J. X. (2015). A host-microbiome interaction mediates the opposing effects of omega-6 and omega-3 fatty acids on metabolic endotoxemia.

Scientific Reports, 5, 11276;

doi: 10.1038/srep11276 (2015).

16 - Doebeli, M., \& Hauert, C. Models of co-operation based on the Prisoner's Dilemma and the Snowdrift game. Ecology Letters, 8(7), 748-766; doi: 10.1111/j.1461-0248.2005.00773.x (2005).

17 - Gore, J., Youk, H., \& Van Oudenaarden, A. Snowdrift game dynamics and facultative cheating in yeast. Nature, 459, 253-256; doi:10.1038/nature07921 (2009).

18 - Hofbauer, J., \& Sigmund, K. Evolutionary games and population dynamics. Cambridge University Press.

ebook ISBN-13: 9781316044377 (1998).

19 - Doebeli, M., Hauert, C., \& Killingback, T. The evolutionary origin of cooperators and defectors. Science, 306, 859-862;

doi: 10.1126/science.1101456 (2004).

20 - Fu, F., Nowak, M. A., \& Hauert, C. Invasion and expansion of co-operators in lattice populations: Prisoner's dilemma vs. snowdrift games. Journal of Theoretical Biology, 266(3), 358-366;

doi: 10.1016/j.jtbi.2010.06.042 (2010).

21 - Bienick, M. S., Young, K.W., Klesmith, J.R., Detwiler, E.E., Tomek, K.J., \& Whitehead, T.A. The interrelationship between promoter strength, gene expression, and growth rate. PLoS One. 9(10), e109105; doi: 10.1371/journal.pone.0109105. eCollection (2014).

22 - Borisova, E., Plamenova, L., Keremedchiev, M., Vladimirov, B., and Avramov, L., Endogenous and exogenous fluorescence of gastrointestinal tumors: initial clinical observations, Proc. SPIE 8770, 
17th International School on Quantum Electronics: Laser Physics and Applications, 87701C; doi: 10.1117/12.2016389 (2013).

23 - Korolev, K. S., Xavier, J. B., Nelson, D. R., and Foster, K. R., A Quantitative Test of Population Genetics Using Spatiogenetic Patterns in Bacterial Colonies, The American Naturalist, 178(4), 538-552; doi: 10.1086/661897 (2011).

24 - Lee, S. M., Donaldson, G. P., Mikulski, Z. Boyajian, S., Ley, K. Mazmanian, S. K. Bacterial colonization factors control specificity and stability of the gut microbiota, Nature, 501(7467), 426-429; 10.1038/nature12447 (2013).

25 - Hickley, C., Sheehan, J. J., Wilkinson, M. G., \& Auty, M. A. E., Growth and location of bacterial colonies within dairy foods using microscopy techniques: a review, Frontiers in Microbiology, 6, 99; doi: 10.3389/fmicb.2015.00099 (2015).

26 - Zheng, D. F., Yin, H. P., Chan, C. H., \& Hui, P. M. Cooperative behavior in a model of evolutionary snowdrift games with $\mathrm{N}$-person interactions. EPL (Europhysics Letters), 80(1), 18002; doi: 10.1209/0295-5075/80/18002 (2007).

27 - Souza, M. O., Pacheco, J. M., \& Santos, F. C. Evolution of cooperation under N-person snowdrift games. Journal of Theoretical Biology, 260(4), 581-588; doi: 10.1016/j.jtbi.2009.07.010 (2009). 28 - Ford, A. C., Quigley, E. M., Lacy, B. E., Lembo, A. J., Saito, Y. A., Schiller, L. R., Soffer, E. E., Spiegel, B. M., \& Moayyedi, P., Efficacy of prebiotics, probiotics, and synbiotics in irritable bowel syndrome and chronic idiopathic constipation: Systematic review and meta-analysis. Am J Gastroenterol. 109(10), 1547-1561; doi: 10.1038/ajg.2014.202 (2014).

29 - Le Barz, M., Anhê, F. F., Varin, T. V., Desjardins, Y., Levy, E., Roy, D., Urdaci, M. C., \& Marette, A., Probiotics as complementary treatment for metabolic disorders. Diabetes \& Metabolism Journal. 39(4): 291-303. doi: 10.4093/dmj.2015.39.4.291 (2015).

30 - Khani, S., Hosseini, H.M., Taheri, M., Nourani, M.R., \& Fooladi, A.A.I., Probiotics as an alternative strategy for prevention and 
treatment of human diseases: a review. Inflammation \& Allergy-Drug Targets (Formerly Current Drug Targets-Inflammation \& Allergy), 11(2), 79-89; doi: 10.2174/187152812800392832 (2012).

31 - Zhang, L. S., \& Davies, S. S. Microbial metabolism of dietary components to bioactive metabolites: opportunities for new therapeutic interventions. Genome Medicine, 8(1), 1-18; doi: 10.1186/s13073-016-0296-x (2016).

32 - Rhim, S. L., Park, M. S., \& Ji, G. E. Expression and secretion of Bifidobacterium adolescentis amylase by Bifidobacterium longum. Biotechnology Letters, 28(3), 163-168; doi: 10.1007/s10529-0055330-9 (2006).

33 - Moya-Pérez, A., Neef, A., \& Sanz, Y. Bifidobacterium pseudocatenulatum CECT 7765 reduces obesity-associated inflammation by restoring the lymphocyte-macrophage balance and gut microbiota structure in high-fat diet-fed mice. PloS One, 10(7), e0126976; doi:10.1371/journal. pone.0126976 (2015).

34 - Hauert, C., De Monte, S., Hofbauer, J., \& Sigmund, K. Replicator dynamics for optional public good games. Journal of Theoretical Biology, 218(2), 187-194; doi: 10.1006/yjtbi.3067 (2002).

35 - Sui, X., Cong, R., Li, K., \& Wang, L. Evolutionary dynamics of N-person snowdrift game. Physics Letters A, 379(45), 2922-2934; doi: 10.1016/j.physleta.2015.08.029 (2015).

36 - Santos, M. D., Pinheiro, F. L., Santos, F. C., \& Pacheco, J. M., Dynamics of $\mathrm{N}$-person snowdrift games in structured populations. Journal of Theoretical Biology, 315, 81-86; doi: 10.1016/j.jtbi.2012.09.001 (2012).

37 - Ohtsuki, H., Pacheco, J. M., \& Nowak, M. A., Evolutionary graph theory: breaking the symmetry between interaction and replacement. Journal of Theoretical Biology, 246(4), 681-694; doi: 10.1016/j.jtbi.2007.01.024 (2007).

38 - Hauert, C., \& Doebeli, M., Spatial structure often inhibits the evolution of co-operation in the snowdrift game. Nature, 428(6983), 643-646; doi: 10.1038/nature02360 (2004). 
39 - Smith, J., The social evolution of bacterial pathogenesis.

Proceedings of the Royal Society of London B: Biological Sciences, 268(1462), 61-69; doi: 10.1098/rspb.2000.1330 (2001).

40 - Nogueira, T., Rankin, D. J., Touchon, M., Taddei, F., Brown, S. P., \& Rocha, E. P., Horizontal gene transfer of the secretome drives the evolution of bacterial co-operation and virulence. Current Biology, 19(20), 1683-1691; doi: 10.1016/j.cub.2009.08.056 (2009).

41 - Mc Ginty, S. E., Rankin, D. J., \& Brown, S. P., Horizontal gene transfer and the evolution of bacterial co-operation. Evolution, 65(1), 21-32; doi: 10.1111/j.1558-5646.2010.01121.x (2011).

42 - Fox, R. E., Zhong, X., Krone, S. M., \& Top, E. M., Spatial structure and nutrients promote invasion of IncP-1 plasmids in bacterial populations. The ISME Journal, 2(10), 1024-1039; doi: 10.1038/ismej.2008.53. (2008).

43 - Baquero, F., Coque, T. M., \& de la Cruz, F., Ecology and evolution as targets: the need for novel eco-evo drugs and strategies to fight antibiotic resistance. Antimicrobial Agents \& Chemotherapy, 55(8), 3649-3660; doi:10.1128/AAC.00013-11 (2011).

44 - Stearns, S. C., Evolutionary medicine: its scope, interest and potential. Proceedings of the Royal Society of London B: Biological Sciences, 279(1746), 4305-4321; doi: 10.1098/rspb.2012.1326 (2012). 45 - Enriquez-Navas, P. M., Kam, Y., Das, T., Hassan, S., Silva, A., Foroutan, P., Ruiz, E., Martinez, G., Minton. S., Gillies. R. J. \& Gatenby, R. A., Exploiting evolutionary principles to prolong tumor control in preclinical models of breast cancer. Science Translational Medicine, 8(327), 327ra24; doi: 10.1126/scitranslmed.aad7842 (2016).

46 - Gatenby, R. A., Brown, J., \& Vincent, T. Lessons from applied ecology: cancer control using an evolutionary double bind. Cancer Research, 69(19), 7499-7502; doi: 10.1158/0008-5472.CAN-09-1354 (2009).

47 - Brown, S. P., West, S. A., Diggle, S. P., \& Griffin, A. S., Social evolution in micro-organisms and a Trojan horse approach to medical 
intervention strategies. Philosophical Transactions of the Royal Society of London B: Biological Sciences, 364(1533), 3157-3168; doi: doi:10.1098/rstb.2009.0055 (2009).

48 - Ibrahim, A. M. The tragedy of the commons and prisoner's dilemma may improve our realization of the theory of life and provide us with advanced therapeutic ways; ResarchGate; doi: 10.13140/RG.2.1.2327.9842 (2015).

49 - Dong, H., Zhi-Hai, R., \& Tao, Z. Zero-determinant strategy: An underway revolution in game theory. Chinese Physics $B, 23(7)$, 078905; doi: 10.1088/1674-1056/23/7/078905 (2014).

50 - Press, W. H., \& Dyson, F. J. Iterated Prisoner's Dilemma contains strategies that dominate any evolutionary opponent. Proceedings of the National Academy of Sciences, 109(26), 10409-10413; doi: 10.1073/pnas.1206569109, (2012).

51 - Pan, L., Hao, D., Rong, Z. \& Zhou, T. Zero-determinant strategies in iterated public goods game. Scientific Reports, 5; doi: 10.1038/srep13096 (2015).

52 - Hilbe, C., Wu, B., Traulsen, A. \& Nowak, M. A., Co-operation and control in multiplayer social dilemmas. Proceedings of the National Academy of Sciences, 111(46), 16425-16430. doi: 10.1073/pnas.1407887111 (2014).

53 - Adami, C. \& Hintze, A., Evolutionary instability of zerodeterminant strategies demonstrates that winning is not everything. Nature Communications 4: 2193. doi: 10.1038/ncomms3193 (2013).

54 - Hilbe C., Nowak MA, \& Sigmund, K., The evolution of extortion in iterated prisoner's dilemma games. Proceedings of the National Academy of Sciences USA 110: 6913-6918; doi:10.1073/pnas.1214834110 (2013).

55 - Hilbe, C., Röhl, T. \& Milinski, M., Extortion subdues human players but is finally punished in the prisoner's dilemma. Nature Communications, 5; doi: 10.1038/ncomms4976 (2014). 
56 - Stewart, A. J. \& Plotkin, J. B., Extortion and co-operation in the prisoner's dilemma. Proceedings of the National Academy of Sciences USA, 109(26), 10134-10135; doi: 10.1073/pnas.1208087109 (2012).

57 - Stewart, A. J., \& Plotkin, J. B. From extortion to generosity, evolution in the iterated prisoner's dilemma. Proceedings of the National Academy of Sciences USA, 110(38), 15348-15353; doi:10.1073/pnas.1306246110 (2013).

58 - Akin, E. Stable cooperative solutions for the iterated prisoner's dilemma. ArXiv.org: Mathematics. Dynamical Systems

ArXiv: 1211.0969v2. (2013).

59 - Shaiju, A. J., \& Bernhard, P., Evolutionarily robust strategies: two nontrivial examples and a theorem. In Advances in Dynamic Games and Their Applications (pp. 1-19). Birkhäuser Boston; doi.org/10.1007/978-0-8176-4834-3_19 (2009).

60 - Hilbe, C., Nowak, M. A., \& Traulsen, A., Adaptive dynamics of extortion and compliance. PLoS One, 8(11), e77886;

doi:10.1371/journal.pone.0077886 (2013).

61 - Archetti, M., Evolutionary stable anti-cancer therapies by autologous cell defection. Evol Med Public Health,(1), 161-172. doi:10.1093/emph/eot014 (2013). 